\title{
Ultrasonographic salivary gland response to Rituximab in secondary Sjögren's syndrome
}

\author{
Vasilia Iorgoveanu, Madalina Gheorghe, Violeta Bojinca, \\ Cosmin Constantinescu, Andra Balanescu, Violeta Vlad, Florian Berghea, \\ Laura Groseanu, Maria Magdalena Negru, Mihai Abobului, \\ Daniela Opris-Belinski, Ruxandra Ionescu
}

"Sf. Maria" Hospital, "Carol Davila" University of Medicine and Pharmacy, Bucharest, Romania

\begin{abstract}
B cell hyper-reactivity implied in the pathogenesis of Sjögren's syndrome (SS) justifies the therapy that involves B cell depletion. Ultrasound of salivary glands demonstrated alterations during disease progression.

Objective. To evaluate changes in salivary gland parenchyma with ultrasound after rituximab treatment in patients' with secondary SS (sSS).

Methods. 7 patients evaluated at baseline (under treatment with rituximab) and after 6 months, underwent ultrasonography of major salivary gland (submandibular and parotid). Clinical data as dryness, pain and diseases activity evaluated with ESSDAI were registered. Salivary gland ultrasound (SGUS) was performed to asses echogenity (using a semiquantitative score from $0-4$, with improvement defined as an at least 1 point decrease), size of gland and glandular borders.

Results. Of 7 patients, 14\% had clinically detectable bilateral parotid tumefaction at baseline. 6 patients $(85.71 \%)$ showed ultrasonographic alterations at baseline. Parotid parenchyma echostructure improved in $42.85 \%$ of patients versus $14.28 \%$ in control group $(p=0.05)$. At the submandibular glands, both submandibular glands showed changes in $28.57 \%$ of patients, while control group showed no changes. $42.85 \%$ patients showed parotid tumefaction which changed in $66.6 \%$ of patients at 6 months.

Conclusion. Ultrasonography showed improvement in salivary gland echostructure in secondary Sjogren Syndrome with rituximab.
\end{abstract}

Keywords: ultrasonography, salivary glands, secondary Sjögren's Syndrome

\section{INTRODUCTION}

Sjögren's syndrome (SS) is a systemic autoimmune disease with a heterogeneous clinical presentation. Dominant symptoms of SS are xerostomia and xerophtalmia, but many patients also experience other type of symptoms, including chronic fatigue, arthralgia and involvement of organs as skin, lungs, kidneys and the nervous system. Dysfunction of exocrine glands is accompanied by periductal mononuclear infiltration of these glands, mainly by CD4 T cells and B. SS pathogenesis is further illustrated by the presence of autoantibodies directed against SS-A/Ro and/or SS-B /La, elevated levels of rheumatoid factor (RF), hypergammaglobulinemia, elevated levels of Bruton's tyrosine kinase in B cells and a significantly increased risk of Non-Hodgkin B-cell lymphoma, predominantly mucosa-associated lymphoid tissue (MALT) lymphoma $(1,2)$.
The prominent role of B cell hyperactivity in pSS pathogenesis provides a rationale for the use of rituximab, a humanized anti-CD20 monoclonal antibody, to treat this disease.

Consensus on the efficacy of rituximab in SS is currently lacking, but treatment results in several clinical, biological and histological improvements.

To date, no systemic treatment has been proven to significantly change the course of SS (primary or secondary), but two randomized double blind studies showed improvement in fatigue and dryness with rituximab treatment $(3,4)$. The randomized, placebocontrolled trial TEARS (Tolerance and Efficacy of Rituximab in primary Sjogren Syndrome) trial (5) showed improvement on recent onset or systemic SS, fatigue being improved. Despite this, French (TEARS) and British (TRACTISS) (6) phase III RCTs failed to demonstrate an effect on primary 
endpoints based on patient-reported visual analogue scales (VAS). The requirement for new and validated outcome measures for PSS led to the development of the European Sjögren's Syndrome Patient Reported Index (ESSPRI) and a physician-assessed systemic disease activity index (European League Against Rheumatism Sjögren's Syndrome Disease Activity Index (ESSDAI). (7) These are helpful, but certain limitations suggest that additional objective outcome measures/biomarkers would be desirable. Other outcome measures include salivary flow rates, although these are subject to issues of standardization and diurnal variation and histological examination of salivary gland biopsies, which may provide mechanistic information but is invasive.

Salivary gland ultrasound (SGUS) is readily available, non-invasive and shows good sensitivity and specificity for diagnosis of SS. In SS, glandular echogenicity is altered with loss of homogeneity with hypoechoic areas and hyperechoic bands. Also, there might be seen a loss of gland definition and size changes. A single sub study of SGUS in TEARS showed that patients had improvement in glandular echostructure at 24 weeks after rituximab treatment compared to placebo (8).

As far as we now, there is no study regarding treatment efficacy in secondary Sjogren's syndrome.

Objective. Use of ultrasound of salivary glands as response predictor for rituximab treatment in secondary Sjogren's Syndrome (sSS).

\section{METHODS}

Clinical data were recorded and ultrasound of salivary glands was performed at baseline and after 6 months. All patients fullfilled the American-European Consensus criteria (9) and diseases activity was assessed with ESSSDAI. Additional requirements were either the presence of xerostomia or xerophtalmia or biomarkers presence in the past or at presentation of anti-Ro-SSA/SSB antibodies, rheumatoid factor, cryoglobulinemia, hypergammaglobulinemia, beta2-microglobulin; or systemic SS with at least one extraglandular manifestation or parotid gland tumefaction. Exclusion criteria were the presence of
HIV infection, tuberculosis, hepatitis B or C, history of cancer. The control group included patients with sSS to rheumatoid arthritis without rituximab.

\section{SGUS assessment}

We used an Esaote Mylab 75 device equipped with a multifrequency linear probe with a frequency derived form 10-15 MHz. We examined all four major salivary glands with gray scale. SGUS was performed before patients were receiving the infusion with rituximab, then 6 months later. Patient position is supine with neck slightly extended and head turned toward the opposite site of the scanned side. Parotid glands were examined in both 2 perpendicular planes. Submandibular glands were assessed only longitudinal. On each side, the parotid gland was scanned in the retromandibular fossa, anterior to the ear and sternocleidomastoid muscle; submandibular gland was assessed in the posterior part of the submandibular triangle. We used a semiquantitative score derived from 0 to 4 (10). Grade 0 represented the normal homogenous gland; grade 1 the presence of small hypoechoic areas without hyperechoic bands; grade 2 multiple hypoechoic areas measuring $<2 \mathrm{~mm}$ with echogenic bands; grade 3 , multiple hypoechogenic areas measuring 2-6 $\mathrm{mm}$ with hyperechogenic bands; and grade 4, multiple hypoechogenic areas measuring $>6 \mathrm{~mm}$ or multiple calcifications with echogenic bands. The sum of each score per gland was considered for analysis (Fig. 1).

\section{Statistical analysis}

We evaluated compared the rituximab group with control group regarding the SGUS change at baseline and at 6 months. We used descriptive statistics for clinical and biological data.

\section{RESULTS}

7 consecutive patients diagnosed with secondary SS (11) were assessed at baseline and after 6 months of treatment with Rituximab. Mean age was $64.86+/-$ 10.9 (min 52 years, $\max 84$ years). All the patients

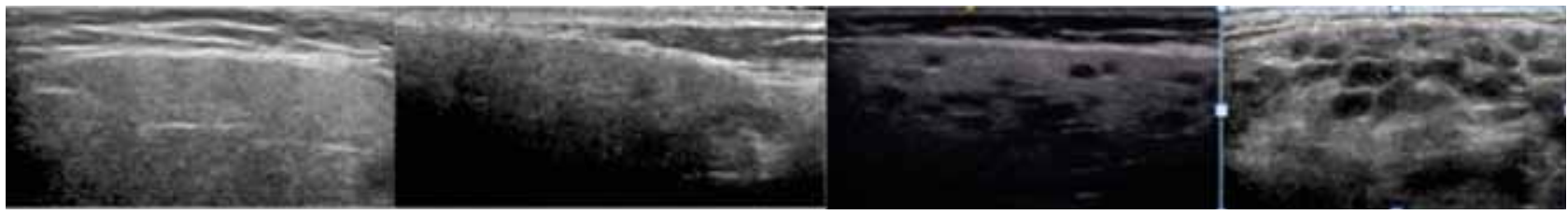

FIGURE 1. Ultrasonographic alterations at parotid gland - score 0-4 
were previously diagnosed with rheumatoid arthritis and treated with Rituximab for 1.71 years $+/ 1.81$ (Table 1).

TABLE 1. General data

\begin{tabular}{|l|c|c|}
\hline \multicolumn{3}{|c|}{ Descriptive statistics } \\
\hline Parameter & Mean (+/- SD) & Range \\
\hline Age (years) & $64.86(+/-10.9)$ & $52-84$ \\
\hline Disease duration (years) & $12.29(+/-5.91)$ & $1-20$ \\
\hline RTX treatment duration & $1.71(+/-1.81)$ & $0-5$ \\
\hline SGUS score & $1.57(+/-1.01)$ & $0-4$ \\
\hline
\end{tabular}

\section{Clinical and serological data}

Disease activity measured with DAS 28 was 3.27 at baseline and reached 2.96 at 6 months (Fig. 2). ESSDAI respected a similar pattern with a decrease or more than 2 points between baseline and followup (Table 2, Fig. 3). All patients experienced xerostomia at both baseline and after 6 months and this level correlated to SGUS score ( $\mathrm{r} 0.24, \mathrm{p}<0.05)$.
During rituximab treatment, DAS 28 has decreased more than in the control group (Table 2). While in control group hematologic changes appeared at follow-up, in the rituximab group no change appeared. Hypocomplementemia returned to normal level in rituximab group at 6 months in $28.57 \%$ of patients. Although both patients and control group did not described improvement in xerostomia, Schirmer test showed a small increase in both groups, with emphasis on rituximab group. Inflammatory syndrome has decreased simultaneously with DAS 28 in both groups, but with higher difference in rituximab group.

4/7 (57.1\%) patients were concomitantly on low dose prednisone at baseline and maintained this dosage during follow-up. 2 patients were taking hydroxychloroquine at baseline and after 6 months, one more patient added. In the control group hydroxychloroquine was continuous. Similar results happen with methotrexate treatment. $14.29 \%$ inter-

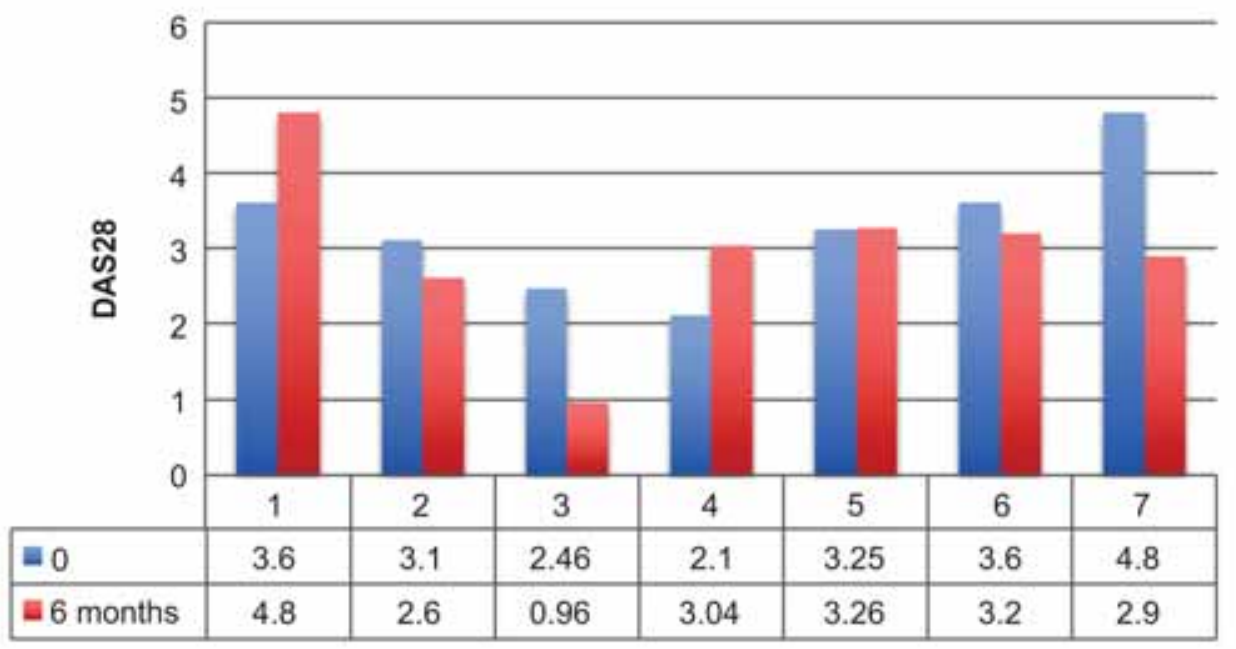

FIGURE 2. Follow up DAS28

TABLE 2. Demographic data

\begin{tabular}{|l|c|c|c|c|}
\hline & Rituximab baseline & Rituximab 6 months & Control baseline & Control 6 months \\
\hline Age & $64.86+/-10.9$ & & $57.85+/-13.14$ & \\
\hline ESSDAl mean, range & $8(0-25)$ & $5.85(0-17)$ & $3.57+/-3.04$ & $3.28+/-2.49$ \\
\hline DAS28 & $3.27+/-0.87$ & $2.96+/-1.13$ & $4.00+/-1.26$ & $3.67+/-0.71$ \\
\hline Articular $\mathrm{n}, \%$ & $4 / 7(57.14)$ & $3 / 7(42.85)$ & $6 / 7(85.71)$ & $6 / 7(85.71)$ \\
\hline Hematologic $\mathrm{n}, \%$ & $1 / 7(14.28)$ & $1 / 7(14.28)$ & $3 / 7(42.85)$ & $2 / 7(28.57)$ \\
\hline Biologic $\mathrm{n}, \%$ & $3 / 7(42.85)$ & $1 / 7(14.28)$ & $0 / 7(0)$ & $0 / 7(0)$ \\
\hline Glandular $\mathrm{n}, \%$ & $1 / 7(14.28)$ & $1 / 7(14.28)$ & $0 / 7(0)$ & $0 / 7(0)$ \\
\hline Vasculitis $\mathrm{n}, \%$ & $2 / 7(28.57)$ & $1 / 7(14.28)$ & $0 / 7(0)$ & $0 / 7(0)$ \\
\hline Schirmer & $7.14+/-3.76$ & $7.42+/-3.55$ & $7+/-4.39$ & $7.14+/-4.22$ \\
\hline Biopsy $\mathrm{n}, \%$ & $2 / 7(28.57)$ & $\mathrm{n} / \mathrm{a}$ & $2 / 7(28.57)$ & $\mathrm{n} / \mathrm{a}$ \\
\hline RF & $71.1+/-60$ & $59,2+/-52$ & $55.83+/-49.23$ & $50.2+/-47.3$ \\
\hline CRP & $10.38+/-7.32$ & $14.14+/-27.84$ & $32.57+/-21.53$ & $23.53+/-35.9$ \\
\hline ESR & $36.71+/-32.58$ & $29.14+/-22.89$ & $32.57+/-21.53$ & $23.53+/-35.9$ \\
\hline SGUS & $(85.7 \%)$ & $4 / 7($ mean $1.12+/-0.99)$ & $2 / 7(\mathrm{mean} 0.28,28.57 \%)$ & $2 / 7(28.57 \%)$ \\
\hline
\end{tabular}




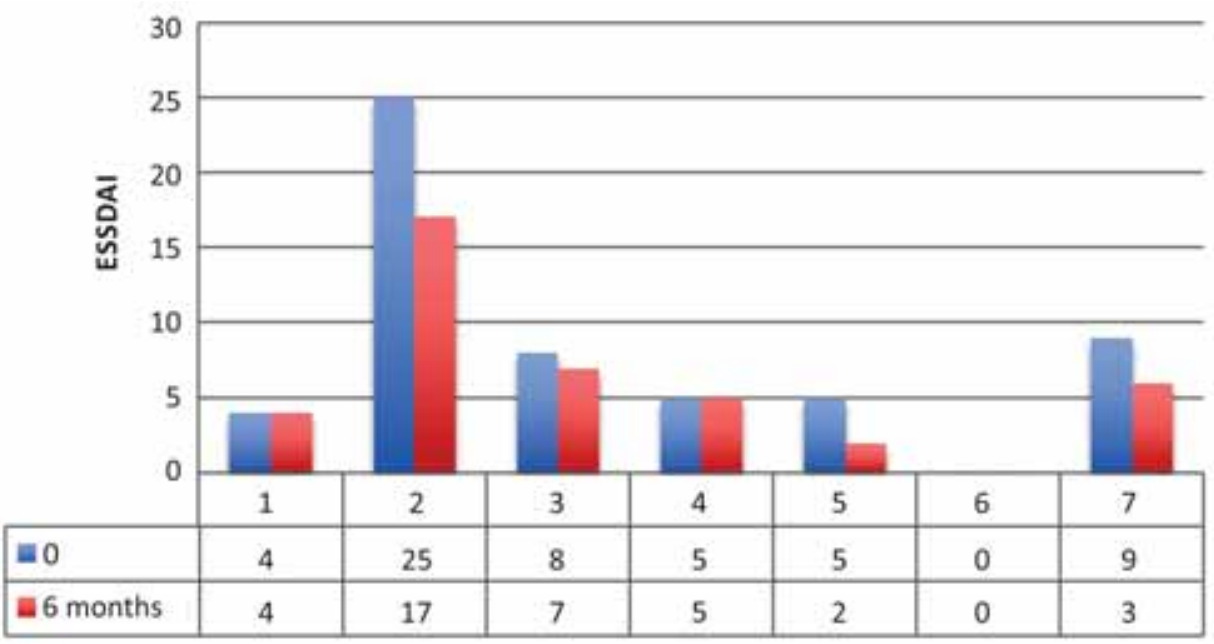

FIGURE 3. Follow up ESSDAI

TABLE 3. Current medication

\begin{tabular}{|l|c|c|c|c|}
\hline \multirow{2}{*}{ Current medication } & \multicolumn{2}{|c|}{ Rituximab group } & \multicolumn{2}{c|}{ Control group } \\
\cline { 2 - 5 } & Baseline & $\mathbf{6}$ months & Baseline & $\mathbf{6}$ months \\
\hline Anticholinergic drugs $\mathrm{n}(\%)$ & $2 / 7(28.57)$ & $1 / 7(14.28)$ & $1 / 7(14.28)$ & $1 / 7(14.28)$ \\
\hline hydroxychloroquine $\mathrm{n}(\%)$ & $2 / 7(28.57)$ & $3 / 7(42.85)$ & $3 / 7(42.85)$ & $3 / 7(42.85)$ \\
\hline Corticotherapy $\mathrm{n}(\%)$ & $4 / 7(57.14)$ & $4 / 7(57.14)$ & $3 / 7(42.85)$ & $3 / 7(42.85)$ \\
\hline Other DMARDs $\mathrm{n}(\%)$ & $4 / 7(57.14)$ & $3 / 7(42.85)$ & $5 / 7(71.42)$ & $5 / 7(71.42)$ \\
\hline Artificial tears $\mathrm{n}(\%)$ & $3 / 7(42.85)$ & $2 / 7(28.57)$ & $3 / 7(42.85)$ & $3 / 7(42.85)$ \\
\hline
\end{tabular}

rupted the administration of other DMARDs compared to control group (none). Artificial tears and anticholinergic drugs were not necessary to use at follow-up for $1 / 3(33.3 \%$ ) patients (Table 3). $3 / 7$ patients underwent treatment for less than a year, average 1.7 years (min-max $0-5$ years).

\section{Ultrasonography data}

SGUS score summed up the score for each gland derived from 0 to 4 describing homogeneity. Mean score was 1.4 at baseline and 1.7 at 6 months distance. 4/7 patients experienced ultrasonographic changes (Fig. 4) during follow-up in rituximab group. Almost all patients have ultrasonographic alterations at baseline $(85.71 \%)$ and $57.14 \%$ of them showed improvement. In the control group, no differences were made between baseline and follow-up.

In the rituximab group, at baseline, most frequent alteration was at left parotid gland with presence of hypoechoic areas under $2 \mathrm{~mm}$ diameter (3/7,

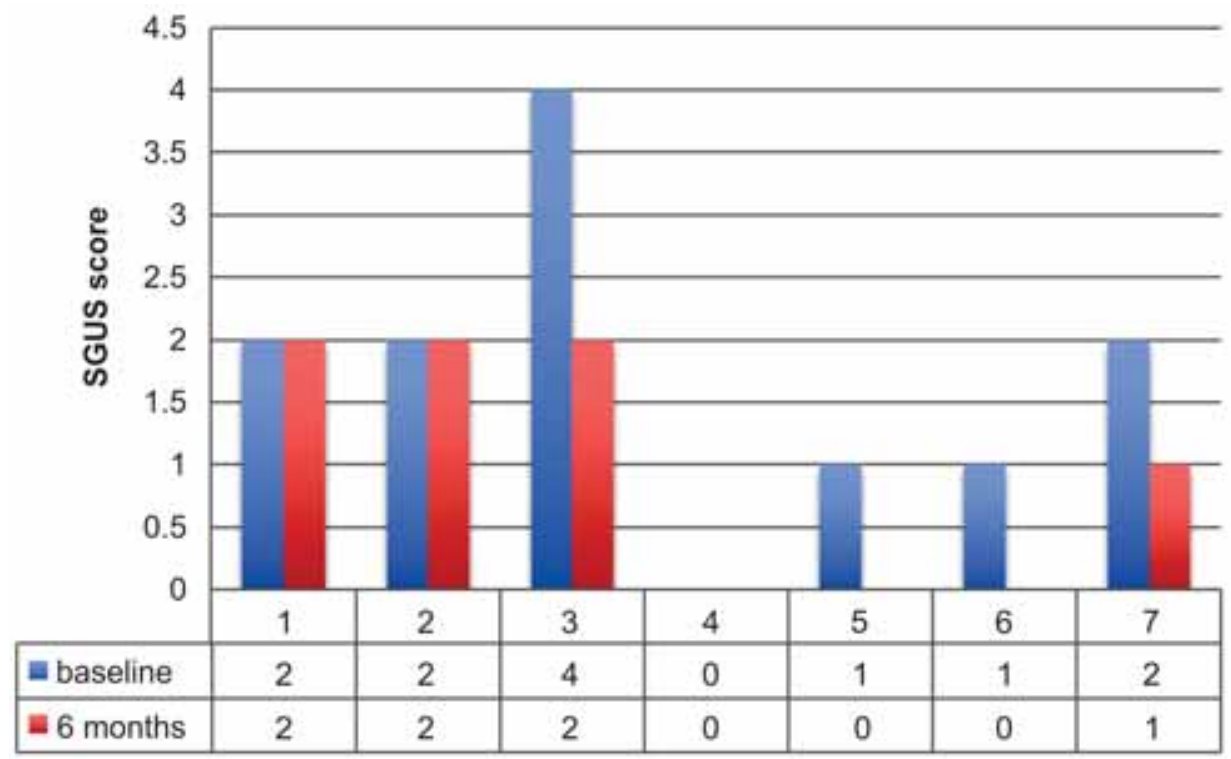

FIGURE 4. Ultrasonographic score during follow-up in rituximab group 
TABLE 4. Ultrasonographic data

\begin{tabular}{|l|c|c|c|c|}
\hline \multirow{2}{*}{ US score/salivary gland } & \multicolumn{2}{|c|}{ Rituximab group } & \multicolumn{2}{c|}{ Control group } \\
\cline { 2 - 5 } & Baseline & $\mathbf{6}$ months & Baseline & $\mathbf{6}$ months \\
\hline Left parotid $\mathrm{n}, \%$ & $4 / 7(57.14)$ & $3 / 7(42.85)$ & $5 / 7(57.14)$ & $5 / 7(57.14)$ \\
\hline Right parotid $\mathrm{n}, \%$ & $3 / 7(42.85)$ & $3 / 7(42.85)$ & $3 / 7(42.85)$ & $3 / 7(42.85)$ \\
\hline Left submandibular $\mathrm{n}, \%$ & $1 / 7(14.28)$ & $0 / 7(0)$ & $2 / 7(28.57)$ & $2 / 7(28.57)$ \\
\hline Right submandibular $\mathrm{n}, \%$ & $2 / 7(28.57)$ & $1 / 7(14.28)$ & $4 / 7(57.14)$ & $4 / 7(57.14)$ \\
\hline
\end{tabular}

$42.85 \%$ ), while in submandibular glands both glands showed minimal alterations $2 / 7$ in right submandibular gland, 1/7 in left submandibular gland). During follow-up, changes were observed in in right submandibular gland and right parotid gland regarding the presence of hypoechoic areas. $3 / 7$ (42.85\%) patients showed parotid tumefaction that changed in $2 / 3(66.6 \%)$ at 6 months. Al glandular borders were conserved at baseline and follow-up (Table 4).

\section{DISCUSSION}

\section{Effects of Rituximab}

Rituximab shows good effects on B cell activity, dryness, fatigue, glandular alterations and several extraglandular manifestations in SS.

The two large studies for efficacy of rituximab treatment may not have met their primary end point for the subjective target and predictors of response. We can also consider further research towards CD-20 immunotherapy or B-cell targeted therapy as anti$\mathrm{CD} 22$ or anti BAFF/Blys antibodies in patients with high disease activity. More, we need more data for treatment efficacy and prevention power for development of extraglandular manifestation and lymphoma.

On B cell activity, several biomarkers of B cell activation (rheumatoid factors, anti SSA, anti SSB, beta 2 microglobulin, free light chains and $\mathrm{B}$ cell activating factor BAFF/Blys) have been studied A small decrease of total serum Ig G after 24 weeks of treatment is seen in large studies (12-14). Dass et al (15) observed that patients with a constant rheumatoid factor level were mostly non-responders to therapy. After B cell repopulation, B cell level rises again and this rise can predict relapse of clinical symptoms.

Similar findings in SSs and rheumatoid arthritis which means combined presence of rheumatoid factors and anti SSA/SSB antibodies resulted in higher disease activity.

In our ultrasonographic findings, there were small differences in parenchymal alteration at baseline and after 6 months of treatment with rituximab.

\section{Predictors of response to rituximab}

In rheumatoid arthritis, RF and anti-ccp antibodies positivity, elevated serum Ig G, low IFN activity, lower serum levels of BAFF and lower circulating levels of plasmablasts were predictors of clinical response to rituximab therapy (16). Responders had a lower focus score and lower salivary gland ultrasonography grade at baseline, compared to non-responders (17).

\section{Clinical efficacy of Rituximab in secondary SS observed with SGUS}

This study provides the first evidence of SGUS changes during treatment with rituximab in sSS. To evaluate the salivary gland echostructure, we choose the simplified De Vita score. No patient showed hyperechoic band of cysts inside the parenchyma. There were defined in majority of cases the presence of hypoechoic areas under $2 \mathrm{~mm}$ and 2-6 $\mathrm{mm}$. The borders of the gland were well defined in both baseline and follow-up. In our study, rituximab treatment was associated with improvement in echostructure exemplified by decrease in number and size of hypoechoic areas compared to control group.

\section{CONCLUSIONS}

Yet, the efficacy of rituximab has not been proven in all patients with SSp, and there is no studies regarding SSs. We need more research towards better definition in primary end points and better inclusion criteria before throwing out this treatment option.

The main limitations of our study are the small number of patients and the baseline definition at different duration of rituximab treatment.

There are studies that suggest adding SGUS score to ACR criteria increases sensitivity. This finding strongly suggests that SGUS may be used as a major tool for monitoring SS outcomes and, most notably, evaluating treatment effects. To sum up, in our small study, SGUS showed improvement in salivary gland echostructure in rituximab group compared to placebo group. 


\section{REFERENCES}

1. Kroese F.G., Abdulahad W.H., Haacke E., Bos N.A., Vissink A., Bootsma H. B-cell hyperactivity in primary Sjogren's syndrome, Expert Rev. Clin. Immunol. 10 (2014) 483-499. doi:10.1586/17446 66X.2014.891439 [doi].

2. Corneth O.B.J., Verstappen G.M.P., Paulissen S.M.J., de Bruijn M.J.W., Rip J., Lukkes M., van Hamburg J.P., Lubberts E., Bootsma H., Kroese F.G.M., Hendriks R.W. Enhanced Bruton's tyrosine kinase activity in peripheral blood B lymphocytes of autoimmune disease patients, Arthritis Reumatol. (2017). doi:10.1002/art.40059.

3. Pijpe J., van Imhoff G.W., Spijkervet F.K., Roodenburg J.L., Wolbink G.J., Mansour K. et al. Rituximab treatment in patients with primary Sjögren's Syndrome. Arthritis Rheum 2005; 52:2740-2750

4. Dass S., Bowman S.J., Vital E.M., Ikeda K., Pease C.T., Hamburger J., Richards A. et al. Reduction of fatigue in Sjögren syndrome with rituximab: results of a randomised, double-blind, placebo-controlled pilot study. Ann Rheum Dis. 2008 Nov; 67(11):1541-4.

5. Devauchelle-Pensec V., Mariette X., Jousse-Joulin S., Berthelot J.M., Perdriger A., Puéchal X. et al. Treatment of Primary Sjögren's Syndrome with Rituximab: A Randomized Trial. An intern med 2014, $160:$ 233-242

6. Bowman S.J., Everett C.C., O'Dwyer J.L. et al. randomized controlled trial of rituximab and cost-effectiveness analysis in treating fatigue and oral dryness in primary sjögren's syndrome. Arthritis Rheumatol 2017; 69:1440-50.

7. Seror R., Theander E., Bootsma H. et al. outcome measures for primary sjögren's syndrome: a comprehensive review. J Autoimmun 2014; 51:51-6.

8. Jousse-Joulin S., Devauchelle-Pensec V., Cornec D. et al. Brief report: ultrasonographic assessment of salivary gland response to rituximab in primary sjögren's syndrome. Arthritis Rheumatol 2015; 67:1623-8.

9. Vitali C., Bombardieri S., Jonsson R. et al. Classification criteria for Sjogren's syndrome: a revised version of the European criteria proposed by the American-European Consensus Group. Ann Rheum Dis 2002; 61:554-8.8. Wernicke D, Hess H, Gromnica-Ihle E, Krause A, Schmidt WA. Ultrasonography of salivary glands a highly specific imaging procedure for diagnosis of Sjögren's syndrome. J Rheumatol. 2008; 35:285-93

10. De Vita S., Lorenzon G., Rossi G., Sabella M., Fossaluzza V. Salivary gland echography in primary and secondary Sjögren's syndrome. Clin Exp Rheumatol. 1992; 10:351-6.

11. Vitali et al. Classification criteria for Sjögren's syndrome: a revised version of the European criteria proposed by the AmericanEuropean Consensus Group. Ann Rheum Dis 2002; 61:554-558.

12. Meijer J.M., Meiners P.M., Vissink A., Spijkervet F.K.L., Abdulahad W., Kamminga N., Brouwer E., Kallenberg C.G.M., Bootsma $\mathrm{H}$. Effectiveness of rituximab treatment in primary Sjögren's syndrome: a randomized, double-blind, placebocontrolled trial, Arthritis Rheum. 62 (2010) 960-8. doi:10.1002/ art.27314.

13. Meiners P.M., Arends S., Meijer J.M. , Moerman R.V., Spijkervet F.K., Vissink A., Bootsma H. Efficacy of retreatment with rituximab in patients with primary Sjogren's syndrome, Clin. Exp. Rheumatol. 33 (2015) 443-444.

14. Devauchelle-Pensec V., Mariette X., Jousse-Joulin S., Berthelot J.M., Perdriger A., Puechal X., Le Guern V., Sibilia J., Gottenberg J.E., Chiche L., Hachulla E., Hatron P.Y., Goeb V., Hayem G., Morel J., Zarnitsky C., Dubost J.J., Pers J.O., Nowak E., Saraux A. Treatment of primary Sjogren syndrome with rituximab: a randomized trial, Ann. Intern. Med. 160 (2014) 233-242.

15. Dass S., Bowman S.J., Vital E.M., Ikeda K., Pease C.T., Hamburger J., Richards A., Rauz S., Emery P. Reduction of fatigue in Sjogren syndrome with rituximab: results of a randomised, double-blind, placebo-controlled pilot study, Ann. Rheum. Dis. 67 (2008) 1541-1544. doi:10.1136/ard.2007.083865.

16. Benucci M., Manfredi M., Puttini P.S., Atzeni F. Predictive factors of response to rituximab therapy in rheumatoid arthritis: What do we know today?, Autoimmun. Rev. 9 (2010) 801-803. doi:10.1016/j.autrev.2010.07.006.

17. Cornec D., Jousse-Joulin S., Costa S., Emery P., Pavitt S. et al. Gray, High-Grade Salivary-Gland Involvement, Assessed by Histology or Ultrasonography, Is Associated with a Poor Response to a Single Rituximab Course in Primary Sjögren's Syndrome: Data from the TEARS Randomized Trial, PLoS One. 11 (2016) e0162787. doi:10.1371/journal.pone.0162787.

18. Cornec D., Jousse-Joulin S., Marhadour T., Pers J.O., Boisramé-Gastrin S., Renaudineau Y. et al. Salivary gland ultrasonography improves the diagnostic performance of the 2012 American College of Rheumatology classification criteria for Sjögren's syndrome. Rheumatology (Oxford). 2014; 53:1604-7

Conflict of interest: none declared Financial support: none declared 